\title{
COLEDOCODUODENOANASTOMOSE NO TRANSPLANTE DE FÍGADO
}

\section{Choledochoduodenostomy in liver transplantation}

\author{
Olival Cirilo de Lucena Neto', Maria Eduarda de Freitas Mesquita do Nascimento², \\ Priscylla Jennie Monteiro Rabêlo1, Paulo Sérgio Vieira de Meloํ․, Américo Gusmão Amorim¹,2, \\ Claudio Moura Lacerda ${ }^{1,2}$
}

\section{RESUMO}

Objetivo: Este trabalho tem o objetivo de descrever cinco casos de coledocoduodenostomia para reconstrução biliar no transplante hepático, suas indicações e seus desfechos. Metódos: Trata-se de estudo retrospectivo dos prontuários de pacientes submetidos a transplante de fígado, entre 1999 e 2019, na Unidade de Transplante de Fígado de Pernambuco (UTF-HUOC), tendo sido selecionados cinco pacientes submetidos a coledocoduodenostomia para reconstrução das vias biliares. Resultados: Os motivos que indicaram a coledocoduodenostomia foram: via biliar do enxerto dilatada e anomalia de via biliar principal; varizes de mesentério; peritonite encapsulante acompanhada de varizes de mesentério e intensas aderências abdominais em dois pacientes. Dentre os cinco pacientes incluídos, dois foram a óbito por disfunção primária do enxerto. Nenhum dos cinco pacientes apresentou fístula biliar e, apenas, um paciente apresentou estenose biliar. Conclusão: Os dados levantados neste trabalho e na literatura apontam para uma semelhança entre Hepaticojejunostomia em $Y$ de Roux e Coledocoduodenostomia em termos de morbidade e mortalidade, com a Coledocoduodenostomia apresentando como vantagem a possibilidade de acesso através de endoscopia.

Descritores: Transplante de Fígado; Ductos Biliares; Procedimentos Cirúrgicos do Sistema Biliar

\footnotetext{
Instituições:

${ }^{1}$ Serviço de Cirurgia Geral e Transplante de Fígado do Hospital Universitário Oswaldo Cruz (HUOC)

2 Universidade de Pernambuco (UPE)
}

\section{Correspondência:}

Olival Cirilo Lucena da Fonseca Neto

Rua Arnóbio Marques, 310, Santo Amaro, CEP:50100-130, Recife/PE (81) 3184-1360

olivalneto@globo.com

\section{INTRODUÇÃO}

A primeira coledocoduodenostomia foi realizada em 1888, por Ridedel, sem sucesso. Já em 1891, o cirurgião alemão Otto Sprengel conseguiu realizar a primeira operação de sucesso utilizando essa técnica, que foi usada no reparo ductal depois de estenose ampular, atresia biliar e coledocolitiase..$^{1,2}$

As complicações biliares continuam sendo uma importante causa de morbimortalidade no transplante hepático e o tipo de anastomose biliar utilizado é um dos fatores que determinam o risco de desenvolver essas complicações. A escolha por uma determinada técnica é influenciada por vários fatores, como a doença base que motivou o transplante, tamanho do ducto biliar do doador e do receptor, retransplante, cirurgias prévias e preferência do cirurgião. Dentre as várias técnicas 
descritas, podemos citar a coledococoledocostomia (CCD), a hepaticojejunostomia em $Y$ de Roux (HJYR) e a coledocoduodenostomia (CDD). A técnica mais comumente utilizada quando o receptor apresenta ducto biliar saudável e de calibre compatível com o doador é CCD, seguida da HJRY, quando não é possível realizar diretamente a anastomose dos ductos biliares ou quando há risco de doença biliar, mas não existe consenso na literatura sobre a melhor abordagem para a reconstrução. ${ }^{1-3}$

No transplante hepático, a CDD foi abandonada após relatos de sepse e ruptura da anastomose e síndrome de Sump em alguns pacientes, apesar da informação limitada para se definir a real incidência e severidade das complicações. Entretanto, experiências com a técnica demonstraram bons desfechos em diversos tipos de reconstruções biliares, sugerindo que essa abordagem poderia ser benéfica ao transplante hepático e novos relatos na literatura demonstraram sua segurança quando comparada às demais técnicas. ${ }^{2,4-6}$

Este trabalho tem o objetivo de descrever cinco casos de coledocoduodenostomia para reconstrução biliar no transplante hepático, suas indicações e seus desfechos (tempo de hospitalização, tempo de follow-up, presença de fístula biliar e de estenose biliar), realizados em um serviço de referência especializado em transplante hepático (Unidade de Transplante de Fígado -UTF) do Hospital Universitàrio Oswaldo Cruz -UPE.

\section{MÉTODOS}

Trata-se de estudo retrospectivo, realizado na Unidade de Transplante de Fígado (UTF) a partir de dados contidos no prontuário dos pacientes que realizaram transplante de fígado entre os anos de 1999 e 2019, tendo sido selecionados cinco pacientes que foram submetidos à coledocoduodenostomia para reconstrução biliar, dentre os 1.400 transplantes realizados no período. Foram avaliadas as seguintes variáveis: idade, sexo, doença base motivadora do transplante, tempo de isquemia quente e fria, tempo de hospitalização, presença de fístula e estenose biliar, técnica cirúrgica, relato de cirurgia prévia, tempo de follow-up e indicação da coledocoduodenostomia.

\section{RESULTADOS}

A amostra selecionada foi de cinco pacientes adultos, sendo dois do sexo feminino e três do sexo masculino, com idade de 53,6 $\pm 6,88$ anos. Dentre eles, três já haviam passado por cirurgias prévias. A doença base que motivou o transplante (Tabela 1) nos pacientes foi Síndrome de Budd-Chiari, doença hepática alcoólica+hepatocarcinoma, esteatohepatite e lesão iatrogênica de vias biliares em dois pacientes.

Tabela 1: Doenças de base dos pacientes incluidos no estudo

\begin{tabular}{lc}
\hline \multicolumn{1}{c}{ Doença de base } & Percentual dos pacientes \\
\hline Síndrome de Budd-Chiari & $1(20 \%)$ \\
Lesão latrogênica de Vias Biliares & $2(40 \%)$ \\
$\begin{array}{l}\text { Doença Hepática Alcoólica associada a } \\
\text { Hepatocarcinoma }\end{array}$ & $1(20 \%)$ \\
Esteatohepatite & $1(20 \%)$ \\
\hline
\end{tabular}

A técnica adotada no transplante foi convencional em três pacientes e piggyback nos outros dois. O tempo médio de isquemia quente dos pacientes foi de $40 \pm$ 9,48 minutos e de isquemia fria foi $6,82 \pm 13,21$ horas. Os motivos que indicaram a coledocoduodenostomia foram: via biliar do enxerto dilatada e anomalia de via biliar principal; varizes de mesentério; peritonite encapsulante acompanhada de varizes de mesentério; intensas aderências abdominais em dois pacientes.

O tempo de hospitalização dos pacientes variou entre dois e nove dias, com média de 5,6 dias. Dois pacientes foram a óbito em 48 horas por disfunção primária do enxerto. $O$ follow-up dos outros três pacientes foi de dois, três e sete anos. Nenhum dos cinco pacientes apresentou fístula biliar e, apenas, um paciente apresentou estenose biliar (33\%).

Tabela 2: Demonstra variáveis técnicas relevantes, do transplante, sobre o procedimento

\begin{tabular}{lc}
\hline Técnica adotada & $\begin{array}{c}\text { Número de pacientes e tempos } \\
\text { cirúrgicos (Percentual) }\end{array}$ \\
Convencional & $3(60 \%)$ \\
Piggyback & $2(40 \%)$ \\
Estenose Biliar & $1(20 \%)$ \\
Tempo de isquemia fria & $6,82 \pm 13,21$ horas \\
Tempo de isquemia quente & $40 \pm 9,48$ minutos \\
Tempo de hospitalização & $5,6 \pm 3$ dias \\
\hline
\end{tabular}

\section{DISCUSSÃO}

A técnica de reconstrução biliar mais comumente utilizada é a coledococoledocostomia. Quando não é possível realizar essa anastomose, areconstrução bilio-entérica é a segunda opção, comumente realizada utilisando-se 
Olival Cirilo de Lucena Neto, Maria Eduarda de Freitas Mesquita do Nascimento, Priscylla Jennie Monteiro Rabêlo, Paulo Sérgio Vieira de Melo, Américo Gusmão Amorim, Claudio Moura Lacerda

a HJYR; entretanto, esse procedimento é associado a complicações como colangite e sangramento gastrointestinal (em cirróticos e abdome com múltiplas reabordagens). A outra opção de reconstrução bilioentérica, utilizada nos pacientes da amostra descrita, é a CDD. ${ }^{3,5}$

A síndrome de sump é uma das possíveis complicações relatadas nas anastomoses coledocoduodenais (láterolateral), sendo um dos fatores que fizeram a HJYR ter preferência sobre a CDD no ambiente cirúrgico, apesar de ser um procedimento mais complexo e demorado. Sua patofisiologia foi teorizada como sendo causada pela estase de bile e refluxo dos componentes duodenais, causando colangite ou abscesso hepático. Apesar do temor da síndrome, a prevalência reportada varia entre 0 e $9,6 \%$, e em um estudo mais recente, $2,5 \%$, sendo uma afecção relativamente rara, que não foi devidamente estudada e esclarecida, bem como as demais complicações da CDD, pela limitada experiência com a técnica reportada na literatura. ${ }^{2,6}$

No transplante de fígado a anastomose coledocoduodenal é realizada de modo términolateral, via biliar distal do enxerto, com uma abertura lateral no duodeno, propiciando ao cirurgião uma anastomose com diametro calibrado. Comparandose a morbidade das duas técnicas, as evidências não sugerem diferença significativa entre a HJYR e a CDD, inclusive em pacientes pediátricos. A CDD apresenta como vantagem a possibilidade de acesso através da endoscopia, reduzindo a necessidade de cirurgias adicionais e drenagem externa. Além disso, apresenta execução mais fácil do que a HJYR em pacientes com cirurgias prévias, como três dos pacientes estudados neste trabalho. Outra vantagem da CDD é apresentar risco significativamente menor de sangramento gastrointestinal quando comparada à HJYR, sendo uma complicação que pode necessitar de cirurgia adicional.

\section{CONCLUSÃO}

Os dados levantados neste trabalho e na literatura apontam para uma semelhança entre HJYR e CDD em termos de morbidade e mortalidade, com a CDD apresentando como vantagem a possibilidade de acesso através da endoscopia. Entretanto, existe deficiência de estudos na literatura sobre o tema, especialmente de estudos randomizados e com coortes significativas, gerando dificuldade em se obter um adequado nível de evidência. Portanto, a utilização da coledocoduodenoanastomose no transplante de fígado só deve ser utilizada em situações de exceção. São necessários mais estudos sobre técnicas de reconstrução biliar no transplante de fígado.

\section{ABSTRACT}

Purpose: This paper aims to describe five choledochoduodenostomy cases for biliary reconstruction in liver transplant. Methods: This is a retrospective study performed from medical records of patients submitted to liver transplant procedure between 1999 and 2019 at Unidade de Transplante de Fígado de Pernambuco (UTF-HUOC), with the selection of five patients who underwent choledochoduodenostomy for biliary reconstruction. Results: The reasons for Choledocoduodenostomy was: dilated graft biliary tract and main biliary tract disorder; mesenteric varices, peritonite encapsulant with mesenteric varices and intense abdominal adherences in two patients. Among the patients included, two died of primary graft disorder. None of the patients presented biliary fistula, and only one presented biliary stenosis. Conclusion: Data observed in this paper and in the literature points to a similarity between Roux-en-Y Choledochojejunostomy and Choledochoduodenostomy in terms of morbidity and mortality, with Choledochoduodenostomy presenting the advantage of possible endoscopic access.

Keywords: Liver Transplantation; Bile Ducts; Biliary Tract Surgical Procedures. 


\section{REFERÊNCIAS}

1. Chok KSH, Lo CM. Systematic review and meta-analysis of studies of biliary reconstruction in adult living donor liver transplantation: Biliary reconstruction in adult LDLT. ANZ J Surg. 2017(mar);87(3):121-5

2. Bennet W. et al. Choledochoduodenostomy is a Safe Alternative to Roux-en-Y Choledochojejunostomy for Biliary Reconstruction in Liver Transplantation. World J Surg. 2009 maio;33(5):1022-5

3.Paes-Barbosa FC, et al. Systematic review and metaanalysis of biliary reconstruction techniques in orthotopic deceased donor liver transplantation. J Hepatobiliary Pancreat Sci. 2011 julho;18(4):525-36
4. Okamoto $\mathrm{H}$. et al. Current assessment of choledochoduodenostomy: 130 consecutive series. Ann R Coll Surg Engl. 2017 set;99(7):545-9.

5. Campsen J. et al. Choledochoduodenostomy in pediatric liver transplantation. Pediatr Transplant. 2011 maio;15(3):237-9.

6. Leppard WM, et al. Choledochoduodenostomy: Is It Really So Bad? J Gastrointest Surg. 2011 maio;15(5):754-7 